\title{
Downside Risk Aversion and the Downside Risk Premium $^{1}$
}

\author{
Richard C. Stapleton ${ }^{2}$ and Qi Zeng ${ }^{3}$
}

${ }^{1}$ We thank C. Gollier (the editor), three anonymous referees, N. Trech, H.Schlesinger, A. Snow, and the participants in the 39th EGRIE annual seminar for helpful comments.

${ }^{2}$ University of Manchester, email:richard.stapleton1@btopenworld.com, Tel: +44 1524 381172

${ }^{3}$ The University of Melbourne, email: qzeng@unimelb.edu.au, Tel: +61383445359

This is the author manuscript accepted for publication and has undergone full peer review but has not been through the copyediting, typesetting, pagination and proofreading process, which may lead to differences between this version and the Version of Record. Please cite this article as doi: 10.1111/jori.12241 


\begin{abstract}
We search for a definition of the downside risk premium analogous to the PrattArrow definition of the risk premium. However, even in the local analysis difficulties arise. To overcome these, we propose a definition based on the difference between two gambles. Further, a global analysis reveals that higher order terms affect the downside risk premium and these cannot be ignored. We show that all five measures of the intensity of downside risk aversion that have been suggested are invalid in the case of the global analysis.
\end{abstract}




\section{Introduction}

Menezes, Geiss and Tressler (1980, hereafter MGT) define increasing downside risk, and show that a downside risk averse agent is characterized by the condition $u^{\prime \prime \prime}(x)>$ 0. Since their initial contribution, numerous attempts have been made to characterize the intensity of downside risk aversion. In the literature there are no fewer than five different measures of the intensity of downside risk aversions, all with strong economic motivations. $^{1}$

In this paper, we investigate how these measures of the intensity of downside risk aversion relate to the downside risk premium. The downside risk premium is the amount that a downside risk averse agent would pay to avoid the downside risk. In the risk aversion analysis by Arrow (1965) and Pratt (1964), the close link between risk aversion and the risk premium plays a critical role in terms of understanding the risk aversion measure. Similarly we will define a downside risk premium which we argue reflects the downside risk aversion of agents. Then we will use this downside risk premium concept to investigate the five measures of downside risk aversion that have been proposed in the literature.

In the original MGT paper, increasing downside risk is defined using a mean-variance preserving transformation (MVPT) between two risky bets. One risky bet is defined

\footnotetext{
${ }^{1}$ Kimball (1990) shows that prudence $-u^{\prime \prime \prime}(x) / u^{\prime \prime}(x)$ explains precautionary saving, Modica and Scarsini $(2005)$ show that $u^{\prime \prime \prime}(x) / u^{\prime}(x)$ is related to the local premium for skewness, Huang (2000) shows that the cautiousness measure $u^{\prime \prime \prime}(x) u^{\prime}(x) / u^{\prime \prime}(x)^{2}$ explains the demand for options. Keenan and Snow (2002) propose the Schwarzian derivative, $S(x)=D(x)-\frac{3}{2} u^{\prime \prime 2}(x) / u^{\prime 2}(x)$ and Liu and Meyer (2012) advocate the use of the rate of change of risk aversion: $\left(-u^{\prime \prime}(x) / u^{\prime}(x)\right)^{\prime}$.
} 
as having more downside risk than the other. In this paper, we compare the downside risk of risky bets. This is in contrast to the definition of risk in Arrow (1965) and Pratt (1964), where a risky bet is compared with a certain payoff. The definition of the downside risk premium in this paper is thus associated with the two risky bets.

The foremost task for any downside risk premium definition is to ensure that, for a downside risk averse agent, namely one with $u^{\prime \prime \prime}(x)>0$, the risk premium is positive. More importantly, by using local analysis, we may associate the downside risk premium with one or more of the measures of downside risk aversion. Specifically, we wish to show that, for two agents with different downside risk aversion, the more downside risk averse agent will pay a higher downside risk premium to avoid the risk. Finally, we would like to show that, globally, the relationship still holds. This is analogous to the process spelled out in the Pratt (1964) analysis of risk aversion and the associated risk premium.

Having introduced our definition of the downside risk premium using the difference between two risky bets, we show that, locally, the risk premium is closely associated with the measure of downside risk aversion proposed in Modica and Scarsini (2005) and Crainich and Eeckhoudt (2008): $D \equiv u^{\prime \prime \prime}(x) / u^{\prime}(x)$. Using local analysis, we also show why it is difficult to define the risk premium explicitly using only one risky bet.

In the following section we use our definition to investigate the global case. Here we find that the above measure does not fit nicely with the risk premium. Furthermore, using the example of the HARA utility function, we show that, in the case of all five suggested measures of downside risk aversion, the link between downside risk aversion 
and the downside risk premium is violated for some numerical examples. Of course, one possible reason is that our definition of the downside risk premium is not the best definition. However, we argue that an alternative similar definition does not work either. ${ }^{2}$ More importantly, we argue in the later discussion section that the source of the problem is more likely to be found in the higher-order derivatives of the utility function, whose effect is not easy to disentangle from the effect of the third-order derivative.

In the remainder of the paper, we first define the downside risk premium and compare it with the monetary compensation proposed in Crainich and Eeckhoudt (2008). Then, using local analysis, we discuss the difficulty which arises when the downside risk premium is linked to just one risky bet. Using the difference between the premiums of two bets, we then link our definition of the downside risk premium to the above-defined $D(x)$. This is followed by a global analysis of the five measures of downside risk aversion. Here, we use the HARA utility function as a counter-example to show that none of the measures consistently predict the premium. Finally we discuss the remaining problems with our analysis and conclude. All the detailed proofs are in the online appendix.

\footnotetext{
${ }^{2}$ Crainich and Eeckhoudt (2008) suggest a premium which they term 'the monetary compensation'. In tests (not reported here) this definition also failed.
} 


\section{Definition of Downside Risk Premium}

MGT characterize an increase in downside risk as a MVPT. An equivalent characterization is proposed by Eeckhoudt and Schlesinger (2006) and Crainich and Eeckhoudt (2008). In this characterization, an independent zero-mean bet $\tilde{\varepsilon}$ is added to a twooutcome bet. It is shown that a downside risk averse agent prefers the case where the bet $\tilde{\varepsilon}$ is added to the branch with the higher value to the one where it is added to the branch with the lower value. Specifically, starting with a bet $\tilde{\eta}$ with two outcomes $\tilde{\eta}=\left(k_{u}, k_{d}\right), k_{u}>k_{d}$, with equal probabilities, an agent with utility function $u(x)$ has the following equivalence in her preferences:

$$
\frac{1}{2} E u\left(x+k_{u}+\tilde{\varepsilon}\right)+\frac{1}{2} u\left(x+k_{d}\right)>\frac{1}{2} u\left(x+k_{u}\right)+\frac{1}{2} E u\left(x+k_{d}+\tilde{\varepsilon}\right) \Longleftrightarrow u^{\prime \prime \prime}(x)>0, \forall x, \tilde{\varepsilon} .
$$

Here the expectation is taken with respect to $\tilde{\varepsilon}$. In Eeckhoudt and Schlesinger (2006) and Crainich and Eeckhoudt (2008), $\tilde{\eta}=\left(k_{u}, k_{d}\right)=(0,-k), k>0$. Here we use the more general format to show that the generic difference between the two risky bets is the downside risk difference, not the particular two-outcome bet that the agent starts with. Note that the two risky bets $\tilde{\eta}_{u} \equiv\left(k_{u}+\tilde{\varepsilon}, k_{d}\right)$ and $\tilde{\eta}_{d} \equiv\left(k_{u}, k_{d}+\tilde{\varepsilon}\right)$ have the same mean and variance ${ }^{3}$. Also, one can show that the transformation from $\tilde{\eta}_{u}$ to $\tilde{\eta}_{d}$ is a MVPT.

Our goal is to define a suitable downside risk premium to characterize the agent's downside risk aversion. First, we define the standard Pratt-Arrow risk premium

\footnotetext{
${ }^{3}$ The variance of the sum of both bets is equal to $\sigma_{\eta}^{2}+\sigma^{2}$, where $\sigma_{\eta}^{2}$ is the variance of the existing risk $\tilde{\eta}$ and $\sigma^{2}$ is the variance of the additional risk, $\tilde{\varepsilon}$.
} 
associated with the two risky bets $\tilde{\eta}_{u}$ and $\tilde{\eta}_{d}$. These are $\pi_{u}$ and $\pi_{d}$ in

$$
\begin{aligned}
u\left(x-\pi_{u}\right) & \equiv \frac{1}{2} E u\left(x+k_{u}+\tilde{\varepsilon}\right)+\frac{1}{2} u\left(x+k_{d}\right) \\
u\left(x-\pi_{d}\right) & \equiv \frac{1}{2} u\left(x+k_{u}\right)+\frac{1}{2} E u\left(x+k_{d}+\tilde{\varepsilon}\right) .
\end{aligned}
$$

$\pi_{u}$ and $\pi_{d}$ are the two risk premiums that the agent is prepared to pay to avoid each of the risky bets $\tilde{\eta}_{u}, \tilde{\eta}_{d}$. From classical risk aversion and risk premium analysis we know that risk aversion plays a critical role in determining the value of risk premiums. Here, though, the discussion of the downside risk premium emphasizes the difference between the two risky bets. Since the two risky bets have the same mean and variance, the difference between the choice of the two risks comes solely from the downside risk aversion of the agent. This leads to the following possible definition of the downside risk premium:

$$
\pi \equiv \pi_{d}-\pi_{u}
$$

Here, the downside risk premium is defined as the difference between the risk premiums of the two risks $\tilde{\eta}_{u}$ and $\tilde{\eta}_{d}$. Of course in comparing the difference between the preferences for $\tilde{\eta}_{u}$ and $\tilde{\eta}_{d}$, there are other ways to define the downside risk premium, each with its own economic meaning. In the above definition, the risk premiums $\pi_{u}$ and $\pi_{d}$ reflect the cost of avoiding the overall risks $\tilde{\eta}_{u}$ and $\tilde{\eta}_{d}$, both which are the sum of the original risk $\tilde{\eta}=\left(k_{u}, k_{d}\right)$ and $\tilde{\varepsilon}$. Implicitly, we are assuming here that both the original risk $\tilde{\eta}=\left(k_{u}, k_{d}\right)$ and $\tilde{\varepsilon}$ are marketable. In other words, there are insurance 
contracts provided to cover the two risks. ${ }^{4} \quad 5 \quad 6$

Before we move on to analyse the relationship between the downside risk premium and downside risk aversion, we want to point out the relationship between our definition of the downside risk premium and the compensation concept employed in Crainich and Eeckhoudt (2008). Crainich and Eeckhoudt propose the following: Since a downside risk averse agent prefers $\tilde{\eta}_{u}$ to $\tilde{\eta}_{d}$, we can define the monetary compensation $m$ given to the agent defined by the equation

$$
\frac{1}{2} E u(x+\tilde{\varepsilon})+\frac{1}{2} u(x-k) \equiv \frac{1}{2} u(x+m)+\frac{1}{2} E u(x-k+\tilde{\varepsilon}), \quad k>0 .
$$

The monetary compensation $m$ is shown to be closely linked to the measure $D(x)=$

\footnotetext{
${ }^{4}$ Actually, we are just asking the following question: if the risks were marketable, how much would the agent be prepared to pay to avoid the combined risks?

${ }^{5}$ Alternatively, suppose that the original risk $\tilde{\eta}=\left(k_{u}, k_{d}\right)$ is fixed and uninsurable. Then $\tilde{\eta}$ is a background risk and we need to consider the derived utility for $x$, given the background risk. In this case, the derived utility function is$$
v(x) \equiv \frac{1}{2} u\left(x+k_{u}\right)+\frac{1}{2} u\left(x+k_{d}\right) .
$$

Then we can define the following two risk premiums associated with the bets $\tilde{\eta}_{u}$ and $\tilde{\eta}_{d}$ :

$$
\begin{aligned}
& v\left(x-\pi_{u, v}\right) \equiv \frac{1}{2} E u\left(x+k_{u}+\tilde{\varepsilon}\right)+\frac{1}{2} u\left(x+k_{d}\right) \\
& v\left(x-\pi_{d, v}\right) \equiv \frac{1}{2} u\left(x+k_{u}\right)+\frac{1}{2} E u\left(x+k_{d}+\tilde{\varepsilon}\right) .
\end{aligned}
$$

We can then further define the downside risk premium as

$$
\pi_{v} \equiv \pi_{d, v}-\pi_{u, v} .
$$

In this definition, the agent has the background risk $\left(k_{u}, k_{d}\right)$ and we ask the following question: What is the difference between the amounts the agent would be prepared to pay to avoid the two risky bets, $\tilde{\eta}_{u}$ and $\tilde{\eta}_{d}$ ? In the subsequent analysis we will look at both of the above definitions of the downside risk premium.

${ }^{6}$ We thank Harris Schlesinger for this suggestion.
} 
$u^{\prime \prime \prime}(x) / u^{\prime}(x)$. The downside risk premium $\pi$ (or $\pi_{v}$ ) defined above and the compensation $m$ both capture the dollar value to the agent of the difference between the two different risky bets. Our definition above is closer to the standard risk premium concept used in the literature.

\section{Local Analysis}

We start by studying the relationship between the downside risk premium and downside risk aversion, as defined in the literature, for example by Modica and Scarsini (2005) and Crainich and Eeckhoudt (2008). Downside risk aversion for agent $i$ is $D(x)=u^{\prime \prime \prime}(x) / u^{\prime}(x)$ and, as in these papers, we start with a local analysis of small risks, where we can accurately approximate the risk premium.

Consider an agent who starts with wealth $x$. Given a scaled random bet $k \tilde{\varepsilon}$ such that $E(\tilde{\varepsilon})=0$, and $k>0$ is some positive constant. The standard Arrow-Pratt risk premium $\pi(k)$ is defined as the amount of money that the agent would be prepared to pay in order to avoid the bet. It is defined by $\pi$ in

$$
u(x-\pi(k)) \equiv E[u(x+k \tilde{\varepsilon})] .
$$

Taking the Taylor expansion on both sides of (6), it follows that

$$
\pi \equiv \pi_{A}+\pi_{D}+o\left(k^{4}\right)
$$


where

$$
\begin{aligned}
\pi_{A} & \equiv \frac{1}{2} A(x) \sigma_{k \epsilon}^{2} \\
\pi_{D} & \equiv-\frac{1}{6} D(x) s_{k \epsilon}^{3},
\end{aligned}
$$

and

$$
\begin{aligned}
& A(x) \equiv-\frac{u^{\prime \prime}(x)}{u^{\prime}(x)} \geq 0 \\
& D(x) \equiv \frac{u^{\prime \prime \prime}(x)}{u^{\prime}(x)}>0 .
\end{aligned}
$$

$A(x)$ and $\pi_{A}$ are the absolute risk aversion and its associated risk premium, i.e. $\pi_{A}$ would be the risk premium if skewness were zero. Then, $\pi_{D}$ is the natural choice for the downside risk premium, and $D(x)>0$ is a measure of downside risk aversion. Given this definition we find:

- $\partial \pi_{D} / \partial s^{3}<0$ : This says that, when the skewness is reduced (i.e. left skewness increases), the downside risk premium increases. This is consistent with the original MGT result. They show that, comparing two bets with the same means and variances, a downside risk averse agent with $u^{\prime \prime \prime}(x)>0$ will prefer the bet with less downside risk. They also show that the bet with less downside risk also has smaller skewness.

- $\partial \pi_{D} / \partial D(x)=-\frac{1}{6} s^{3}$ : Hence, if skewness is negative, $s^{3}<0$, then an increase in $D(x)=u^{\prime \prime \prime}(x) / u^{\prime}(x)$ increases the downside risk premium. However, if skewness 
is positive, $s^{3}>0$, then an increase in $D(x)=u^{\prime \prime \prime}(x) / u^{\prime}(x)$ reduces the downside risk premium.

Consider two agents $i, j$ with the same risk aversion, $A^{i}(x)=A^{j}(x)$, but with one being more downside risk averse than the other, indicated by $D^{i}(x)>D^{j}(x)$. If they both face a bet with downside risk, indicated by $s^{3}<0$, then indeed $\pi_{D}^{i}>\pi_{D}^{j}>0$. Both agents dislike this bet and will pay more cash in addition to $\pi_{A}$ to avoid the bet. The higher is $D(x)$, the more the agent dislikes the downside risk.

However, if the two agents face a bet with "upside risk", indicated by $s^{3}>0$, $\pi_{D}^{i}<\pi_{D}^{j}<0$. Now again both agents will pay $\pi_{A}$ to avoid the risk, but the fact that $s^{3}>0$ means that the upside skewed distribution mitigates the risk itself, $\pi_{D}<0$. Now higher downside risk aversion $D(x)$ means that the agent will appreciate the additional bet more.

The second point above is not surprising given the discussion in the introduction: Increasing downside risk is closely associated in MGT with a MVPT between two risky bets. A properly defined downside risk premium should reflect this difference between the two risks. So we propose the following two risk premium definitions:

$$
\begin{aligned}
& u\left(x-\pi_{u}\right) \equiv \frac{1}{2} E u\left(x+k_{u}+\tilde{\varepsilon}\right)+\frac{1}{2} u\left(x+k_{d}\right), \\
& u\left(x-\pi_{d}\right) \equiv \frac{1}{2} u\left(x+k_{u}\right)+\frac{1}{2} E u\left(x+k_{d}+\tilde{\varepsilon}\right) .
\end{aligned}
$$


One can show that the premium $\pi_{u}$ is approximately

$$
\pi_{u}=-\frac{k_{u}+k_{d}}{2}+\frac{1}{4} A(x)\left(k_{u}^{2}+k_{d}^{2}+\sigma^{2}\right)-\frac{1}{12} D(x)\left(k_{u}^{3}+k_{d}^{3}+3 k_{u} \sigma^{2}+s^{3}\right),
$$

where $A(x)$ and $D(x)$ are as defined above. Here the first term is just the mean value of $\tilde{\eta}_{k}$. If $k_{u}=-k_{d} \equiv k>0$, it is zero. The second term is the risk premium associated with the total risk, indicated by the variance of $\tilde{\eta}_{k}$ plus $\tilde{\varepsilon}$. The third term is related to the downside risk. As discussed above, the sign of this term is determined by the skewness of both $\tilde{\eta}_{k}$ and $\tilde{\varepsilon}$.

Similarly, from (13), we find that the premium $\pi_{d}$ is approximately

$$
\pi_{d}=-\frac{k_{u}+k_{d}}{2}+\frac{1}{4} A(x)\left(k_{u}^{2}+k_{d}^{2}+\sigma^{2}\right)-\frac{1}{12} D(x)\left(k_{u}^{3}+k_{d}^{3}+3 k_{d} \sigma^{2}+s^{3}\right) .
$$

Thus the downside risk premium, defined as the difference between the premium on the lower branch and the premium on the higher branch, is given by

$$
\pi=\pi_{d}-\pi_{u} \approx \frac{1}{4} D(x)\left(k_{u}-k_{d}\right) \sigma^{2}>0 .
$$

When $k_{u}=-k_{d}=k>0$, we have $\pi=\frac{1}{2} D(x) k \sigma^{2}>0$. When $k_{u}=0, k_{d}=-k<0$, $\pi=\frac{1}{4} D(x) k \sigma^{2}>0$.

In the introduction we suggested that the downside risk premium should satisfy two criteria. First, given $u^{\prime \prime \prime}(w)>0$, it should be positive. (16) confirms that this is the case. Second, given two agents $i$ and $j$ who are downside risk averse, and 
$D_{i}(x)>D_{j}(x)$, we should have $\pi_{i}>\pi_{j} \cdot{ }^{7}$

\subsection{Downside Risk Premium when $\tilde{\eta}$ is Non-tradable}

So far we have derived the downside risk premium for a risk $\tilde{\varepsilon}$ together with an existing risk $\tilde{\eta}$, implicitly assuming that both risks are tradable. We now consider the case where the existing risk $\tilde{\eta}$ is non-tradable and has to be treated as a background risk. In this case, the downside risk premium is determined by the derived utility function $v(x)=E[u(x+\tilde{\eta})]$. In the case of the two-state existing risk:

$$
\begin{aligned}
v(x) & =E[u(x+\tilde{\eta})] \\
& =\frac{1}{2} u\left(x+k_{u}\right)+\frac{1}{2} u\left(x+k_{d}\right) .
\end{aligned}
$$

Our proposed definition of the downside risk premium in this case is similar to that in the case where the existing risk is tradable. The difference is that the derived downside risk aversion is defined by

$$
D_{v}(x) \equiv \frac{v^{\prime \prime \prime}(x)}{v^{\prime}(x)}
$$

\footnotetext{
${ }^{7}$ Also, we should note that, using this risk premium to characterize downside risk aversion also performs well in relation to the concerns raised by Liu and Meyer (2012). It is a transitive, partialorder relationship.
} 
When both $\tilde{\eta}$ and $\epsilon$ are small, the downside risk premium is hence given by

$$
\pi=\frac{1}{4} D_{v}(x)\left(k_{u}-k_{d}\right) \sigma^{2}>0
$$

where

$$
D_{v}(x) \equiv \frac{v^{\prime \prime \prime}(x)}{v^{\prime}(x)}=\frac{u^{\prime \prime \prime}\left(x+k_{u}\right)+u^{\prime \prime \prime}\left(x+k_{d}\right)}{u^{\prime}\left(x+k_{u}\right)+u^{\prime}\left(x+k_{d}\right)} .
$$

When the background risk is small, we can simplify the above expression further using an approximation. Then the downside risk premium becomes:

$$
\pi=\frac{1}{4} D_{v}(x)\left(k_{u}-k_{d}\right) \sigma^{2}>0
$$

The definitions of the downside risk premium in this paper, both in the case where the existing risk $\eta$ is tradable and when it is not, are closely related to previous suggested definitions in Keenan and Snow (2009), Modica and Scarsini (2005) and Crainich and Eeckhoudt (2008). Keenan and Snow (2009), for example, introduce 'a differential risk premium, compensating for an increase in risk' and add, 'one cannot introduce downside risk, one can only change the downside risk of a risk that is already present' ${ }^{\prime 8}$. Modica and Scarsini also use an approximation approach and define a differential premium

$$
\pi_{x}-\pi_{y} \approx \frac{1}{6} D\left[E\left(x^{3}\right)-E\left(y^{3}\right)\right]
$$

\footnotetext{
${ }^{8}$ Keenan and Snow (2009, p.1098).
} 
Crainich and Eeckhoudt use a similar approach and define a 'monetary compensation' $m$ :

$$
m \approx \frac{\sigma^{2}}{2} k \frac{u^{\prime \prime \prime}(x)}{u^{\prime}(x)}
$$

\subsection{Downside Risk Premium and Asset Pricing}

In this section we relate our definition of downside risk premium to the returns of securities that have been studied extensively in the finance literature ${ }^{9}$. More specifically we will show that our definition of downside risk premium, and the local measure $D(x)$ defined above, is relevant for theories of the risk free rate and expected cross-sectional stock returns used in the finance literature.

It is well-known that the risk free rate is closely related to the impatience and the consumption growth of agents in the economy (Ramsey Rule). Under uncertain consumption growth and power utility, the risk-free rate is also affected by agents' precautionary savings motive. Gollier (2013) illustrates this extended Ramsey rule. In the following, we will use a variation of a two-period model in Gollier (2003) to study the risk free rate and cross-sectional return relationships in a complete market, and link our definition of the downside risk premium to them.

Consider an economy with two dates, $t=0,1$. Agents in this economy maximize a time-additive utility function by trading a set of securities, indexed by i. Denote price and the (possibly risky) payoff of security $i$ as $P_{i}, D_{i}$ respectively. The agents'

\footnotetext{
${ }^{9}$ We thank Christian Gollier for pointing out this extension.
} 
problem is:

$$
\max _{\Theta_{i}} u\left(C_{0}\right)+e^{-\delta} E\left[u\left(C_{1}\right)\right]
$$

such that

$$
\begin{aligned}
W_{0} & =C_{0}+\sum_{i} \Theta_{i} P_{i} \\
C_{1} & =W_{1}+\sum_{i} \Theta_{i} D_{i} .
\end{aligned}
$$

Here, $W_{0}, W_{1}$ are the endowment of an agent at time $t=0,1$, and $e^{-\delta}$ is the subject discount factor of the agent. For simplicity we assume the set of the securities is such that the market is complete. Then the first order condition gives us the price for any security as a function of marginal utility of an agent and the payoff of the security:

$$
P_{i}=e^{-\delta} E\left[\frac{u^{\prime}\left(C_{1}\right)}{u^{\prime}\left(C_{0}\right)} D_{i}\right], \quad \forall i
$$

Let us start with the risk free bond, whose payoff $D_{i}$ is constant. The risk free rate, defined as $1+R_{f} \equiv D_{f} / P_{f}$, is given by:

$$
1+R_{f}=e^{\delta}\left\{E\left[\frac{u^{\prime}\left(C_{1}\right)}{u^{\prime}\left(C_{0}\right)}\right]\right\}^{-1}
$$

Following Gollier (2013) and Martin(2012), define the continuous compounded return as:

$$
r_{f} \equiv \ln \left(1+R_{f}\right)=\delta-\ln E\left[\frac{u^{\prime}\left(C_{1}\right)}{u^{\prime}\left(C_{0}\right)}\right]
$$


Under the assumption of power utility, $u(C)=C^{1-\gamma} /(1-\gamma)$, the above risk-free rate can be approximated as:

$$
\begin{aligned}
r_{f} & \approx \delta+\gamma \frac{E\left(C_{1}\right)-C_{0}}{C_{0}}-\frac{1}{2} \operatorname{var}\left(\frac{E\left(C_{1}\right)-C_{0}}{C_{0}}\right) \gamma(\gamma+1) \\
& =\delta+\frac{-C_{0} u^{\prime \prime}\left(C_{0}\right)}{u^{\prime}\left(C_{0}\right)} \frac{E\left(C_{1}\right)-C_{0}}{C_{0}}-\frac{1}{2} \operatorname{var}\left(\frac{E\left(C_{1}\right)-C_{0}}{C_{0}}\right) \frac{C_{0}^{2} u^{\prime \prime \prime}\left(C_{0}\right)}{u^{\prime}\left(C_{0}\right)} .
\end{aligned}
$$

As pointed out in Gollier (2013), the precautionary savings motive comes from the agents' aversion to the uncertainty of the consumption growth in the future. That agents are downside risk averse implies that $u^{\prime \prime \prime}(c)>0$. This enhances the incentive for the agents to save, thus agents do not need the resulting risk-free rate to be as high to induce them to save. The magnitude of the reduction in risk-free rate is exactly equal to the downside risk premium we defined above under the situation $k_{u}=-k_{d}=1$.

The relationship between the downside risk aversion and the risk-free rate is through the convexity of the marginal utility of the agents. One might argue that the effect of the precautionary savings motive is still through agents' aversion to the riskiness of their own consumption growth. For example, if the variance of the consumption growth rate increases by one percent, the risk free rate will reduce by half a percentage point multiplying by the relative downside risk aversion intensity. We next show that our definition of the downside risk premium from the difference between two risky bets is also closely related to the cross-sectional return research.

The study of this cross-sectional stock returns has been a central topic in financial 
research ever since the development of Capital Asset Pricing Model (CAPM) during 1960s. The Consumption-based CAPM (CCAPM) aims to give a more fundamental source of the pricing kernel, which is a function of the aggregate consumption growth. The risk premium of any security is the expected excess return of the security required to induce the representative agent with risky consumption growth to hold such a security.

Using the above setup, we define the expected return of any security as $1+E R_{i} \equiv$ $E\left(D_{i}\right) / P_{i}$. Again we use continuously compounded return and make use of the price function (26):

$$
\begin{aligned}
r_{i} & \equiv \ln \left(1+E R_{i}\right) \\
& =\ln E\left(D_{i}\right)+\delta-\ln E\left[\frac{u^{\prime}\left(C_{1}\right)}{u^{\prime}\left(C_{0}\right)} D_{i}\right]
\end{aligned}
$$

The risk premium of the asset is given by:

$$
r p_{i} \equiv r_{i}-r_{f}
$$

For power utility, the above risk premium is given by:

$$
r p_{i}=\ln E\left(D_{i}\right)+\ln E\left[\left(\frac{C_{1}}{C_{0}}\right)^{-\gamma}\right]-\ln E\left[\left(\frac{C_{1}}{C_{0}}\right)^{-\gamma} D_{i}\right]
$$

Motivated by Martin (2012), we work on the log consumption growth and log divi- 
dend. Define (we omit the subscript i for now)

$$
\begin{aligned}
& g_{C} \equiv \ln \frac{C_{1}}{C_{0}}, \\
& g_{D} \equiv \ln D .
\end{aligned}
$$

The above risk premium can be written as:

$$
r p=\ln E\left(e^{g_{D}}\right)+\ln \left(e^{-\gamma g_{C}}\right)-\ln \left(e^{-\gamma g_{C}+g_{D}}\right) .
$$

As in Martin(2012), we introduce the two-variate cumulant-generating function (cgf) as:

$$
c\left(\theta_{1}, \theta_{2}\right) \equiv \ln \left(e^{\theta_{1} g_{C}+\theta_{s} g_{D}}\right)
$$

Then the risk premium can be conveniently written as:

$$
r p=c(0,1)+c(-\gamma, 1)-c(-\gamma, 1)
$$

One can approximate this expression to the third order ${ }^{10}$ :

$r p=-\gamma \operatorname{cov}\left(g_{C}, g_{D}\right)-\frac{1}{2} E\left(\left(g_{C}-E\left(g_{C}\right)\right)^{2}\left(g_{D}-E\left(g_{D}\right)\right)\right) \gamma^{2}+\frac{1}{2} E\left(\left(g_{C}-E\left(g_{C}\right)\right)\left(g_{D}-E\left(g_{D}\right)\right)^{2}\right) \gamma$

The last two terms are simply the co-skewness between $g_{C}$ and $g_{D}$. And this holds for any risky security.

\footnotetext{
${ }^{10}$ The derivation is the same as in Martin (2012) and available upon request.
} 
Using this approximation we can calculate and compare the risk premiums of two risky assets which are otherwise identical, but one has more downside risk than the other. For example, we can assume that the states of the world are spanned by two random variables $\tilde{\eta} \times \tilde{\epsilon}$. The first is a binary distribution with equal probability and values being \pm 1 . The second random variable has value $\tilde{\epsilon}$ which is conditional independent from $\tilde{\eta}$ with mean zero and variance $\sigma^{2}$. Denote the conditional cdf of $\tilde{\epsilon}$ to be $p_{\epsilon}$.

Now we assume that the $\log$ aggregate consumption growth $g_{C}= \pm 1+\epsilon$ with probability density $\frac{1}{2} p_{\epsilon}$. And there are two risky securities with log payoff given by:

$$
\begin{aligned}
& g_{u}= \begin{cases}2 \epsilon & , \text { when } \eta=1 \\
0 & , \text { when } \eta=-1\end{cases} \\
& g_{d}=\left\{\begin{array}{cl}
0, & \text { when } \eta=1 \\
2 \epsilon, & \text { when } \eta=-1 .
\end{array}\right.
\end{aligned}
$$

Using the above expression for the risk premium of any security, the difference between the risk premiums of the two assets is given by:

$$
\pi \equiv r p_{d}-r p_{u}=\gamma(\gamma+1) \sigma^{2}
$$

where $\gamma(\gamma+1)$ is $C^{2} U^{\prime \prime \prime}(C) / U^{\prime}(C)$.

This definition of difference in the cross-sectional risk premiums is very common in the asset pricing literature. The most well-known ones are the value premium 
and size premium studied by Fama and French (1993). The value premium is the difference between the risk premium of the value stocks (stocks with high book-tomarket ratio) and that of the growth stocks (stocks with low book-to-market ratio). The size premium is the difference between the risk premium of the small stocks and that of the large stocks. Our definition of downside risk premium also follow this line of research by comparing the difference in risk premiums between two risky bets, where one has more downside risk than the other.

\section{Global Analysis: Comparison of Measures Pro- posed in the Literature}

Motivated by the local analysis, we use the downside risk premium defined above to evaluate the five measures of downside risk which have been proposed in the literature. We now no longer assume that $\varepsilon$ is restricted to be small. The first measure of downside risk is prudence, $P(x)=-u^{\prime \prime \prime}(x) / u^{\prime \prime}(x)$ introduced by Kimball (1990), and subsequently used by Chiu $(2005,2010)$. The second measure is the definition we have used, $D(x)=u^{\prime \prime \prime}(x) / u^{\prime}(x)$, proposed by Modica and Scarsini (2005) and Crainich and Eeckhoudt (2008). The third measure is the Schwarzian derivative, $S(x)=$ $D(x)-\frac{3}{2} u^{\prime 2}(x) / u^{\prime 2}(x)$, proposed by Keenan and Snow $(2002,2009,2010)$. The fourth measure is the rate of change in absolute risk aversion, $A^{\prime}(x)=D(x)-u^{\prime \prime 2}(x) / u^{\prime 2}(x)$, proposed by Liu and Meyer (2012). Finally, there is cautiousness, or the rate of change

of risk tolerance, introduced by Wilson (1968), and used by Huang and Stapleton 
(2014). Cautiousness is $C(x)=P(x) / A(x)-1$. In this section, we investigate whether these measures can answer the question raised at the beginning of the last section. Specifically, we want to see whether it is true that, if an agent is more downside risk averse than another, the downside risk premium required by the former will be higher than that required by the latter.

Here we show using numerical examples that none of the measures can answer the above question consistently. The basic set up of the numerical examples is as follows. We compare two agents with the HARA utility function:

$$
u(x)=\frac{\gamma}{1-\gamma}\left(\frac{x}{\gamma}+b\right)^{1-\gamma}
$$

where $\frac{x}{\gamma}+b>0$. For this function,

$$
u^{\prime \prime \prime}(x)=\frac{(\gamma+1)}{\gamma}\left(\frac{x}{\gamma}+b\right)^{-\gamma-2}>0
$$

if and only if $\gamma>0$ or $\gamma<-1$. Also, given $u^{\prime}(x)>0, u^{\prime \prime \prime}(x)>0$ implies that $D(x)>0$. It follows immediately that the downside risk premium, $\pi$, is positive for the agent.

We now look at whether $D_{i}(x)>D_{j}(x)$ implies that $\pi_{i}>\pi_{j}$ in the global case. Unfortunately, this is not always true. To see why, we consider how $\pi$ depends on $x$ and the parameters $b$ and $\gamma$ in the case of HARA utility. One can show the downside risk premium, defined as above as the difference between $\pi_{d}$ and $\pi_{u}$, is approximated 
by

$$
\begin{aligned}
\pi & \approx \frac{1}{6} \gamma(\gamma+1) \frac{3\left(k_{u}-k_{d}\right) \sigma^{2} / 2}{(x+\gamma b)^{2}} \\
& +\frac{1}{24} \gamma(\gamma+1)(-\gamma-2) \cdot \frac{\left[6\left(k_{u}^{2}-k_{d}^{2}\right) \sigma^{2}+4\left(k_{u}-k_{d}\right) s^{3}\right] / 2}{(x+\gamma b)^{3}}
\end{aligned}
$$

Note that the measure $D(x)=\frac{(\gamma+1)}{\gamma}(x+\gamma b)^{-2}$. We can rewrite therefore the above expression using the $D(x)$ measure:

$$
\pi \approx \frac{1}{4} D(x)\left(k_{u}-k_{d}\right) \sigma^{2}-\frac{1}{24}(\gamma+2) D(x) \frac{3\left(k_{u}^{2}-k_{d}^{2}\right) \sigma^{2}+2\left(k_{u}-k_{d}\right) s^{3}}{x+\gamma b} .
$$

The first term in (47) is just the approximate term we derived for a general (i.e. not necessarily HARA) utility function in the previous local analysis. The second term is determined by the higher-order moments, in this case by the skewness of $\tilde{\varepsilon}$.

Checking the first term alone, one can see that our previous analysis seems to indicate that, for two agents with $D_{1}(x)>D_{2}(x), \pi_{1}>\pi_{2}$. However, the second term shows that this is not necessarily true when we adjust for the higher-order terms. The effect of the higher-order moments can potentially dominate the first term if the denominator $x+\gamma b$ is small enough and this can be the case if $b<0$ and $\gamma b$ is close to $x .{ }^{11}$ We will show later using the numerical example that this is indeed the case.

\footnotetext{
${ }^{11} b<0$ is commonly assumed for the HARA function, since it signifies declining relative risk aversion.
} 
For example, if $k_{u}=-k_{d}=k>0$, the above expression becomes

$$
\pi \approx \frac{1}{2} D(x) k \sigma^{2}-\frac{1}{6}(\gamma+2) D(x) \frac{k s^{3}}{x+\gamma b}
$$

Note that $k s^{3}$ does not depend on $x, b$ and $\gamma$. Thus, when $x+b \gamma$ is small enough the change in the second term can exceed the change in the first term if $s^{3}>0$, resulting in a negative change in $\pi$.

In a similar manner, we can express the premium as a function of each of the other four measures of the downside risk aversion intensity. Under HARA utility these measures are as follows :

$$
\begin{aligned}
P(x) & =(\gamma+1)(x+\gamma b)^{-1} \\
S(x) & =\gamma\left(1-\frac{1}{2} \gamma\right)(x+\gamma b)^{-2} \\
A^{\prime}(x) & =\gamma(x+\gamma b)^{-2} \\
C \equiv C(x) & =\frac{1}{\gamma} .
\end{aligned}
$$

Under the assumption that $k_{u}=-k_{d}=k$, we can rewrite the downside risk premium (47) as:

$$
\pi \approx \frac{1}{2} \gamma(\gamma+1) \frac{k \sigma^{2}}{(x+\gamma b)^{2}}-\frac{1}{6} \gamma(\gamma+1)(\gamma+2) \cdot \frac{k s^{3}}{(x+\gamma b)^{3}}
$$

Thus we can write the downside risk premium as a function of the five measures as 
the following:

$$
\begin{aligned}
\pi & =\frac{1}{2} k \sigma^{2} D(x)-\frac{1}{6}(\gamma+2) \frac{k s^{3}}{x+\gamma b} D(x) \\
& =\frac{\gamma}{2(\gamma+1)} k \sigma^{2} P(x)^{2}-\frac{\gamma(\gamma+2)}{6(\gamma+1)^{2}} k s^{3} P(x)^{3} \\
& =\frac{\gamma+1}{2-\gamma} k \sigma^{2} S(x)-\frac{(\gamma+1)(\gamma+2)}{3(2-\gamma)} \frac{k s^{3}}{x+\gamma b} S(x) \\
& =\frac{\gamma+1}{2} k \sigma^{2} A^{\prime}(x)-\frac{(\gamma+1)(\gamma+2)}{6} \frac{k s^{3}}{x+\gamma b} A^{\prime}(x) \\
& =\frac{1}{2}(1+C) \frac{k \sigma^{2}}{(x C+b)^{2}}-\frac{1}{6}(1+C)(2+C) \cdot \frac{k s^{3}}{(x C+b)^{3}} .
\end{aligned}
$$

The last expression in (58) can be written as:

$$
\pi=\frac{(1+C) k}{(x C+b)^{3}}\left[\frac{1}{2}(x C+b) \sigma^{2}-\frac{1}{6}(2+C) s^{3}\right] .
$$

Note that $x$ is the starting wealth of the agents, $k$ is only related to the initial bet, and $\sigma^{2}, s^{3}$ are only related to the $\epsilon$ that is being allocated to either the upper or down branch of the initial bet.

Assuming $(k, 1 / 2,-k, 1 / 2)$ is independent of $\epsilon$, the following property follows immediately from the above expressions:

- For any given $D(x), P(x), S(x), A^{\prime}(x), C$ at any $(x, k, \gamma, b)$, the effect on the downward risk premium $\pi$ changes sign between $\left(\sigma^{2}>>s^{3}\right)$ and $\left(\sigma^{2}<<s^{3}\right)^{12}$.

\footnotetext{
${ }^{12}$ The notation $\sigma^{2}>>s^{3}$ means that $\sigma^{2}$ is sufficiently greater than $s^{3}$.
} 


\section{Numerical Examples}

We use the following numerical values to show that counter examples exist for all of the measures of downside risk aversion. In other words: for each measure, there exists an example where for two agents, the downside risk aversion measure is larger for one agent than for the other, while the premium demanded is smaller. The general set up for the eamples is the following. We assume $x=20, k_{u}=-k_{d}=5$. An independent $\tilde{\varepsilon}$ is uniformly distributed with integer value from -5 to 5 (11 states with equal probability 1/11). We fix the preference of the first agent as $\gamma_{1}=0.3, b_{1}=1$. We fix $\gamma=0.2$ for the second agent, and let $b=-18$ or $b=-20$.

\begin{tabular}{cccccc}
\hline & Agent 1 & Agent 2 (A) & Difference & Agent 2 (B) & Difference \\
\hline $\mathrm{b}$ & 1 & -18 & & -20 & \\
\hline $\mathrm{P}$ & 0.064 & 0.073 & 0.009 & 0.075 & 0.011 \\
$\mathrm{D}$ & 0.000946 & 0.000892 & $-5.41 \mathrm{E}-05$ & 0.000938 & $-8.9 \mathrm{E}-6$ \\
$\mathrm{~S}$ & 0.000619 & 0.000669 & $5 \mathrm{E}-5$ & 0.000703 & $8.4 \mathrm{E}-5$ \\
$\mathrm{~A}^{\prime}$ & 0.000728 & 0.000744 & $1.6 \mathrm{E}-5$ & 0.000781 & $5.3 \mathrm{E}-5$ \\
$\mathrm{C}$ & 4.333333 & 6 & 1.6667 & 6 & 1.6667 \\
\hline$\pi$ & 0.0264 & 0.0263 & -0.0001 & 0.0279 & 0.0015 \\
\hline
\end{tabular}

In the above Table, we show the results of calculations of the downside risk premium and the five measures of downside risk aversion, assuming investors have HARA utility with either $\gamma=3, b=1$ (investor 1 ) or $\gamma=2, b=-18$, or $b=-20$ (investor 2). The first line shows prudence. Agent 1 has $P=0.064$. Agent 2 with $\gamma=2, b=-18$ 
has $P=0.073$. In the third column, we show the difference in prudence, 0.009. In the final two columns, we show similar results for the case of Agent 2 with $\gamma=2$, $b=-20$. Lines $2,3,4,5$ show similar results for the measures $D, S, A^{\prime}, C$. In the final line of the Table we show the downside risk premium computed using equation (4).

Note first that comparing Agent 2 (with $b=-18$ ) with Agent 1, the change in the measure $\mathrm{D}$ is consistent with the change in the downside risk premium, $\pi$ (both decline for Agent 2). D declines from 0.000946 to 0.000892 , while $\pi$ declines from 0.0264 to 0.0263. On the other hand, all the remaining measures $P, S, A^{\prime}, C$ increase. Hence, using this comparison, we can reject these four measures as invalid for purposes of predicting the downside risk premium.

Now compare Agent 2 (with $b=-20$ ) with investor 1 . Looking at the $D$ measure, we see that $D$ declines from 0.000946 to 0.000938 . However, the downside risk premium increases from 0.0264 to 0.0279 . This example enables us to also reject $D$ as a predictor of the premium.

The computations shown in the Table reveal that all five of the proposed measures of downside risk aversion intensity are rejected as inconsistent predictors of the downside risk premium. This negative result confirms the suspicion of Keenan and Snow, who speculate that none of these measures adequately reflect the premium. 


\section{Ross comments}

In comparing the utility of outcomes in the up-state $u$ and the down-state $d$ and the risk premiums $\pi_{u}$ and $\pi_{d}$ we need to be mindful of the criticisms of Ross (1981). Ross shows that Arrow-Pratt risk aversion can yield incorrect predictions when more than one risky asset are present. ${ }^{13}$ Ross suggests a stronger definition of risk aversion which gives a correct prediction when an agent uses a less risky asset to finance the purchase of a more risky asset. Using 'Ross risk aversion' a more (Ross) risk averse agent invests less in the more risky asset than an identical less (Ross) risk averse agent.

We now consider the relevance of the Ross analysis in the context of downside risk aversion. We have defined the downside risk premium as the difference between $\pi_{d}$ and $\pi_{u}$. However, note that $\pi_{d}$ and $\pi_{u}$ predict how the agent would behave when faced with the choice between a single risky asset and the risk-free asset. Also, the downside risk premium $\pi_{d}-\pi_{u}$ predicts the difference in these behaviors in the down-state $d$ and the up-state $u$. There is always a single risky asset in this discussion. Hence the Ross (1981) analysis and (Ross) risk aversion is neither required or relevant here.

Also, it should be noted that our definition of the downside risk premium is the simple difference between two risk premiums $\pi_{d}$ and $\pi_{u}$. It does not involve the conditional risk premium $\pi_{u / d}$. It has been shown by Doherty, Louberge and Schlesinger (1992) that $\pi_{u} \neq \pi_{d}+\pi_{u / d}$. However, since our definition of the premium does not involve

\footnotetext{
${ }^{13}$ The need for Ross risk aversion when two risky assets are involved is proved and explained in Huang and Litzenberger (1988),sections 2.11-2.14.
} 
$\pi_{u / d}$, this is not an issue here. ${ }^{14}$ Finally, we should note that our definition $\pi_{d}$ $\pi_{u}$ yields a downside risk premium which is identical to that of Modica and Scarsini (2005), which has been accepted by many as one of the most useful definitions of downside risk aversion.

\section{Discussion and Conclusion}

We have established a definition of the downside risk premium as the difference between two risk premiums. This is consistent with the work of Keenan and Snow, Modica and Scarsini, and Crainich and Eeckhoudt and works well in the case of a local analysis. However, all of the measures of downside risk aversion that have been suggested in the literature, fail to give a consistent prediction of the downside risk premium in the global case. We show that this is due to the fact that a third order approximation of the premium fails to capture relevant higher order terms. By using the HARA utility function as a special case we show that it is easy to construct counter examples to the proposition that any of the measures of downside risk aversion capture the effect on the downside risk premium.

\footnotetext{
${ }^{14}$ We thank a referee for raising this point.
} 


\section{REFERENCES}

1. Arrow, K. J. (1965). Aspects of a Theory of Risk Bearing, Yrjo Jahnsson Lectures, Helsinki. Reprinted in Essays in the theory of Risk Bearing (1971). Chicago: Markham Publishing Co.

2. Chiu, W. H. (2000). On the Propensity to Self-protect. Journal of Risk and Insurance 67, 555-578.

3. Chiu, W. H. (2005). Skewness Preference, Risk Aversion, and the Precedence Relations on Stochastic Changes, Management Science 12, 1816-1828.

4. Chiu, W. H. (2010). Skewness Preference, Risk Taking and Expected Utility Maximisation, The Geneva Risk and Insurance Review 35, 108-129.

5. Chiu, W. H. (2012). Risk Aversion, Downside Risk Aversion and Paying for Stochastic Improvements, The Geneva Risk and Insurance Review 37, 1-26.

6. Crainich, D., \& Eeckhoudt, L. (2008). On the Intensity of Downside Risk Aversion, Journal of Risk and Uncertainty 36, 267-276.

7. Denuit, M. M., \& Eeckhoudt, L. (2010). A General Index of Absolute Risk Attitude, Management Science 56, 712-715.

8. Doherty, N., H. Louberge, H. Schlesinger, (1987) Additive and Multiplicative Risk Premiums with Multiple Source of Risk, Scandinavian Actuarial Journal, $1-2,41-49$. 
9. Eeckhoudt, L., \& Schlesinger, H. (1994). A Precautionary Tale of Risk Aversion and Prudence, B. Munier and M. J. Machina (eds.), Models and Experiments in Risk and Rationality, 75-90, Kluwer Academic Publishers. Printed in the Netherlands.

10. Fama, E. \& French, K. (1993). Common risk factors in the returns on stocks and bonds, Journal of Financial Economics 33, 3-56.

11. Galsband, V., (2012). "Downside Risk of International Stock Returns. Journal of Banking and Finance 36, 2379-2388.

12. Gollier, C., (2013), Pricing the Planet's Future: The Economics of Discounting in an Uncertain World., Princeton University Press.

13. Huang, J. (2000). Relationships between Risk Aversion, Prudence, and Cautiousness, Working paper, Lancaster University.

14. Huang, J. (2012). A Note on Comparative Skewness Preferences. Working paper, available at http://ssrn.com/abstract=2190442 or http://dx.doi.org /10.2139/ssrn.2190442.

15. Huang, C, \& Litzenberger, R. H (1988), Foundations for Financial Economics, Elsevier Science Ltd.

16. Huang, J., and R. Stapleton (2014), Cautiousness in the Small and in the Large, Review of Finance 18 2375-2395. 
17. Jindapon, P. (2010). Prudence Probability Premium. Economics Letters 109, $34-37$.

18. Jindapon, P., \& Neilson, W. (2007). Higher-order Generalizations of ArrowPratt and Ross Risk Aversion: A Comparative Statics Approach, Journal of Economic Theory 136, 719-728.

19. Keenan, D., and A. Snow (2002). Greater Downside Risk Aversion, Journal of Risk and Uncertainty 24, 267-277.

20. Keenan, D., and A. Snow (2009). Greater Downside Risk Aversion in the Large, Journal of Economic Theory 144, 1092-1101.

21. Keenan, D., and A. Snow (2010). Greater Prudence and Greater Downside Risk Aversion, Journal of Economic Theory 145, 2018-2026.

22. Keenan, D., \& Snow, A. (2012). The Schwarzian Derivative as a Ranking of Downside Risk Aversion, Journal of Risk and Uncertainty 44, 149-160.

23. Kimball, M. S. (1990). Precautionary Saving in the Small and in the Large. Econometrica 58, 5373.

24. Liu, L., \& Meyer, J. (2012). Decreasing Absolute Risk Aversion, Prudence and Increased Downside Risk Aversion. Journal of Risk and Uncertainty 44, 243-260.

25. Martin, I. (2013), Consumption-Based Asset Pricing with Higher Cumulants, Review of Economic Studies, 80, 745-773. 
26. Modica, S., and M. Scarsini (2005). A Note on Comparative Downside Risk Aversion. Journal of Economic Theory 122, 267-271.

27. Pratt, J. W. (1964). Risk Aversion in the Small and in the Large, Econometrica $32,122-136$.

28. Ross, S. (1981), Some Stronger Measures of Risk Aversion in the Small and in the Large with Applications, Econometrica, 49, 621-638.

29. Van Zwet, W. R. (1964). Convex Transformations of Random Variables, Mathematical Centre Tracts 7, Mathematisch Centrum, Amsterdam.

30. Wilson, R. (1968), The Theory of Syndicates, Econometrica, 36, 119-132. 


\section{University Library}

\section{- M M N E R VA A gateway to Melbourne's research publications}

Minerva Access is the Institutional Repository of The University of Melbourne

Author/s:

Zeng, Q;Stapleton, RC

Title:

Downside Risk Aversion and the Downside Risk Premium

Date:

2018-06-01

Citation:

Zeng, Q. \& Stapleton, R. C. (2018). Downside Risk Aversion and the Downside Risk Premium. Journal of Risk and Insurance, 85 (2), pp.379-395. https://doi.org/10.1111/ jori.12241.

Persistent Link:

http://hdl.handle.net/11343/283439 